\title{
A NOTE ON SOME CHARACTERIZATIONS OF SIDON SETS
}

\author{
RON C. BLEI
}

ABSTRACT. A simple proof of the following theorem is presented: $E \subset Z$ is a Sidon set if and only if $A_{E}(T)$ equals any one of the following three spaces: $U_{E}(T), \bigcap_{p>1} A_{E}^{p}(T)$, and $C_{E}(T, \omega)$.

In this note we present an elementary proof of some characterizations of Sidon sets, announced by K. Ross (joint work with R. E. Edwards and E. Hewitt) at the International Conference on Harmonic Analysis in Maryland, November 1971 (cf. [1]).

We refer to [2] for standard definitions and facts. For $E \subset Z$, let $U_{E}(T)=\left\{f \in C_{E}(T):\left\langle S_{n}(f)\right\rangle\right.$ converges uniformly to $\left.f\right\}$, and let $A_{E}^{p}(T)=$ $\left\{f \in C_{E}(T): \hat{f} \in l^{p}\right\} ;$ for a fixed element $\omega \in c_{0}(E)$, let $A_{E}(T, \omega)=$ $\left\{f \in C_{E}(T): \omega \hat{f} \in l^{1}\right\} . E \subset Z$ is a Sidon set if $A_{E}(T)=C_{E}(T)$. We remark that $E$ is a Sidon set if and only if $\inf \left\{\|f\|_{\infty} /\|f\|_{A}: f \in A_{E}, f \neq 0\right\}>0$.

THEOREM. The following statements are equivalent:

(i) $E$ is a Sidon set,

(ii) $U_{E}(T)=A_{E}(T)$,

(iii) $\bigcap_{p>1} A_{E}^{p}(T)=A_{E}(T)$,

(iv) $A_{E}(T,(1))=A_{E}(T)$.

Proof. (ii), (iii) $\Rightarrow$ (i): Suppose $E \subset Z$ is not a Sidon set. Without loss of generality, we assume $E \subset Z^{+}$. By our preceding remark, we select $\left\langle f_{j}\right\rangle$, a sequence of trigonometric polynomials in $C_{E}(T)$, subject to the following conditions:

(i) $\left\|f_{j}\right\|_{\infty}<1 / 2^{j}$,

(ii) $\left\|f_{j}\right\|_{A}=1 / j$,

(iii) $N_{j} \equiv \min \left\{n: n \in \operatorname{support}\left(\hat{f}_{j}\right)\right\}>\max \left\{n: n \in \operatorname{support}\left(\hat{f}_{i}\right)\right\}$, whenever $i>i$.

Set $f=\sum f_{j}$. Clearly $f \in C_{E}$, and, by (ii), $f \notin A_{E}$.

By (i), $\left\langle S_{N_{i}}(f)\right\rangle$ converges uniformly to $f$. It follows from (ii) that

$$
\begin{aligned}
\left\|S_{N}(f)-f\right\|_{\infty} & =\left\|S_{N_{j}}(f)+\left(S_{N^{\prime}}(f)-S_{X_{j}}(f)\right)-f\right\|_{\infty} \\
& \leqq\left\|S_{X_{j}}(f)-f\right\|_{\infty}+\left\|S_{N^{V}}(f)-S_{X_{j}}(f)\right\|_{\infty} \\
& \leqq\left\|S_{X_{j}}(f)-f\right\|_{\infty}+1 / j,
\end{aligned}
$$

Received by the editors February 17, 1972 and, in revised form, March 10, 1972. AMS 1969 subject classifications. Primary 4212. 
where $j$ is selected so that $N_{j}<N<N_{j+1}$. Therefore, $f \in U_{E}$. Since $\left\|f_{j}\right\|_{A} \geqq$ $\left\|\hat{f}_{j}\right\|_{p}$, and $\sum\left\|\hat{f}_{j}\right\|_{.1}<\propto$ for all $p>1$, we have $f \in \bigcap_{p>1} A_{E}^{p}$.

(iv) $\Rightarrow$ (i). Let $\left\langle i_{j}\right\rangle$ be so that $\omega_{k}<1 / j$ for all $k \geqq i_{j}$. Select $f_{j}$ as above, with the additional requirement that $N_{j} \geqq i_{j}$. It easily follows that $\omega \hat{f} \in l^{1}$. The theorem is proved.

REMARK. The proof of (iii), (iv) $\Leftrightarrow$ (i) can easily be adapted to general compact abelian groups.

\section{REFERENCES}

1. R. E. Edwards, E. Hewitt and K. A. Ross, Lacunarity for compact groups. II, Pacific J. Math. (to appear).

2. J.-P. Kahane, Séries de Fourier absolument convergentes, Ergebnisse der Mathematik und ihrer Grenzgebiete, Band 50, Springer-Verlag, Berlin and New York, 1970. MR 43 \#801.

Department of Mathematics, University of Connecticut, Storrs, Connecticut 06268 